\title{
PSFC/JA-06-15
}

\section{Sawtooth period changes with mode conversion current drive on Alcator C-Mod}

\author{
A. Parisot, S. J. Wukitch, P. T. Bonoli, M. Greenwald, \\ A. Hubbard, Y. Lin, R. Parker, M. Porkolab, J.C. Wright
}

June 2006

\author{
Plasma Science and Fusion Center \\ Massachusetts Institute of Technology \\ Cambridge MA 02139 USA
}

This work was supported by the U.S. Department of Energy, Cooperative Agreement No. DE-FC02-99-ER54512. Reproduction, translation, publication, use and disposal, in whole or in part, by or for the United States government is permitted.

Submitted for publication to Plasma Physics and Controlled Fusion. 


\title{
Sawtooth period changes with mode conversion current drive on Alcator C-Mod
}

\author{
A. Parisot, S.J. Wukitch, P. Bonoli, M. Greenwald, A. \\ Hubbard, Y. Lin, R. Parker, M. Porkolab, A.K. Ram, J.C. \\ Wright
}

Plasma Science and Fusion Center, MIT, Cambridge MA 02139 USA

\begin{abstract}
.
Significant changes in the sawtooth period have been observed on the Alcator CMod tokamak during phased ICRF operation in the mode conversion regime. As the mode conversion layer was swept outward through the $\mathrm{q}=1$ surface in $\mathrm{D}\left({ }^{3} \mathrm{He}\right)$ plasmas, the sawtooth period was found to increase and then decrease for counter-current drive phasing. For co-current drive and heating phasings, it was observed to decrease and then increase. With $2 \mathrm{MW}$ ICRF power, the period varied from 3 to $12 \mathrm{~ms}$. The observed evolution is consistent with localized current drive by mode converted waves in the vicinity of the $q=1$ surface. Simulations with the full wave code TORIC indicate that the electron heating and current drive is due to mode converted Ion Cyclotron Waves. The observed evolution for symmetric (heating) phasing is difficult to attribute to localized heating, since temperature profile stiffness prohibits large changes in the resistivity gradient at the $\mathrm{q}=1$ surface. An alternative explanation is found in TORIC simulations, which predict co-current drive for symmetric phasing due to a strong updown asymmetry in the ICW wave field.
\end{abstract}

E-mail: parisot@mit.edu 


\section{Introduction}

In tokamak plasmas with multiple ion species, fast magnetosonic waves excited by radiofrequency antennas in the Ion Cyclotron Range of Frequencies (ICRF) can mode convert to shorter wavelength modes in the plasma core. The mode converted (MC) waves damp in the immediate vicinity of the mode conversion region, which results in strong localized absorption. The relative concentration of the ion species can be adjusted so that the mode conversion region is well separated from the ion cyclotron layers. In this regime cyclotron damping of the fast wave and $\mathrm{MC}$ waves becomes weaker and electron Landau damping of the MC waves dominates. This mode conversion electron heating (MCEH) scheme has been investigated in many tokamak experiments including TFTR [Majeski 96], Tore-Supra [Saoutic 96], ASDEX-Upgrade [Noterdaeme 96], Alcator CMod [Bonoli 97, Lin 03], and JET [Mantsinen 04]. It has proved to be an efficient technique for localized electron heating. The heating location can be controlled by changing the plasma composition or the toroidal magnetic field, and is predicted accurately from the cold plasma dispersion relation of the fast wave in the zero electron mass limit:

$$
n_{\perp}^{2}=-\frac{\left(R-n_{\|}^{2}\right)\left(L-n_{\|}^{2}\right)}{S-n_{\|}^{2}}
$$

where R, L and S are the dielectric tensor elements in the Stix notation [Stix 92] and $n_{\perp}=k_{\perp} c / \omega, n_{\|}=k_{\|} c / \omega$ are the refractive indexes perpendicular and parallel to the magnetic field. The $S=n_{\|}^{2}$ condition defines a resonance surface where $n_{\perp}^{2} \rightarrow \infty$ and therefore possible mode conversion can occur [Swanson 85]. The peak electron heating location was shown to be in good agreement with the midplane location of the mode conversion layer $S=n_{\|}^{2}$ in several MCEH experiments. The power deposition profiles can be predicted using full wave numerical simulations. The models include relevant thermal corrections in the cold plasma dielectric tensor and solve the wave equation in either slab or toroidal geometries. Very good agreement with experimentally measured profiles is generally obtained (see for example [Bonoli 97, Wright 04]). MECH appears therefore as a relatively well understood electron heating scheme, with possible applications to next-step tokamak devices. Experiments in TFTR [Wilson 98] showed that MCEH scenarios can be used in reactor-relevant D-T plasmas, without density limit constraints as in electron cyclotron or neutral beam heating.

Beyond electron heating, the strong interaction with electrons suggests other possible applications like localized current drive and current profile control. The possibility of mode conversion current drive (MCCD) has been demonstrated in TFTR experiments [Majeski 96], however the physics and efficiency of this technique have not been yet investigated in detail. Compared to MCEH, MCCD involves more complicated aspects of mode conversion physics. This stems largely from two effects:

- The strong dependence of the current drive efficiency on the parallel wavenumber $k_{\|}$, particularly for low parallel phase velocity waves. In most MCEH scenarios, mode 
converted waves tend to damp with parallel phase velocities $\frac{\omega}{k_{\|}}$close or below the electron thermal speed, and therefore can interact strongly with trapped particles or close to the trapped-passing boundary. This will reduce the efficiency depending on the damping location and local $k_{\|}$spectrum.

- The evolution of $k_{\|}$in toroidal geometry [Ram 91]. This can be seen from the approximate expression $k_{\|} \approx \frac{n_{\phi}}{R}+\frac{m_{\theta}}{r} \frac{B_{\theta}}{B}$, where $n_{\phi}, m_{\theta}$ are toroidal and poloidal mode numbers respectively and $R, r$ is the local major and minor radius. While $n_{\phi}$ is conserved due to toroidal geometry, $m_{\theta}$ can vary significantly as the perpendicular wavenumber $k_{\perp}$ changes rapidly in the mode conversion region. If the poloidal field is sufficiently large, the $m_{\theta}$ term can dominate and the initial spectrum imparted by the antenna is partly lost.

As a result, the physics of MCCD involves detailed aspects of the mode conversion process and is tied to the propagation characteristics of mode converted waves in toroidal geometry.

This topic has been a major focus of the Alcator C-Mod ICRF program. Full wave simulations in toroidal geometry with the full wave code TORIC [Brambilla 99, Wright 04] have been compared with measured power deposition profiles [Lin 03] and with wave-induced density fluctuations observed by Phase Contrast Imaging (PCI) [Nelson-Melby 03, Lin 05]. Remarkable agreement with the code predictions has been observed on both aspects. The code was able to reproduce the spatial structure and radial spectrum of PCI signals associated with mode converted Ion Bernstein Waves (MCIBW) and with Ion Cyclotron Waves (MCICW). While MCIBW are predicted by slab models, the existence of MCICW in the core of toroidal plasmas is directly related to poloidal-field induced upshifts in $k_{\|}$[Perkins 77, Faulconer 89, Parisot 05]. It has been confirmed by numerical simulations in toroidal geometry [Jaeger 03, Wright 04] and by the experimental observation of wave-induced density fluctuations by PCI signal on the low field side of the ion-ion hybrid layer [Nelson-Melby 03], since MCIBW cannot propagate there. Characteristic structures indicating poloidal asymmetries in the MCICW wavefields were observed both in the simulations and experimental data, which are associated with upshifts and downshifts in $k_{\|}$. This gives good confidence that the essential physics involved in MCCD is now accessible to modeling tools in the Ion Cyclotron Range of Frequencies, and has motivated further MCCD studies on C-Mod, based on TORIC predictions.

This paper reports on MCCD experiments where sawtooth period changes with deposition close to the inversion radius are used as a means to infer localized current drive. A similar approach was used by Bhatnagar et al. [Bhatnagar 94] to study Ion Cyclotron Current Drive (ICCD). Initial experiments [Wukitch 05] in Alcator C-Mod showed significant differences in the sawtooth period between co- and counter-current drive phasings when the mode conversion layer was located just inside the inversion radius. The period was shortened to $5 \mathrm{~ms}$ in co-CD phasing and lengthened to 15 ms in counter-CD phasing. Near axis deposition did not change the sawtooth period 
significantly, suggesting a localized effect. These initial results, together with more recent studies on sawtooth period modeling [Porcelli 96, Graves 05], motivated the experiment reported here, in which the mode conversion surface was swept through the $\mathrm{q}=1$ surface.

Experimental data and initial analysis for two sets of discharges will be presented in this paper. The setup and approach will be discussed in section 2. The sawtooth period evolution during the sweep in co/counter and heating phasing will be presented in section 3. Significant changes in the sawtooth period are observed for co- and counter-current drive phasing, and possible mechanisms for the observed evolution are discussed. We will show that the experimental data is consistent with localized current drive at the mode conversion layer. This is also consistent with numerical predictions with the TORIC code, which will be presented in section 4 . The TORIC simulations show a strong updown asymmetry in the electron damping of the mode converted waves, which results in net current drive in the co-current direction for symmetric phasing. This prediction will be compared with experimental data in section 5. While the data is consistent with the TORIC predictions, localized electron heating from the mode converted waves could also account for the observed evolution. However, the effect of localized heating should be small in these C-Mod discharges due to strong temperature profile stiffness. Conclusions from this experiment will be discussed in section 6 .

\section{Experimental setup}

The Alcator C-Mod device [Hutchinson 93] is a compact (major radius $\mathrm{R}=0.67 \mathrm{~m}$, minor radius $0.21 \mathrm{~m})$, high field $\left(B_{T} \leq 8.1 \mathrm{~T}\right)$ diverted tokamak. It is equipped with three fast wave ICRF antennas [Wukitch 02]. The two 2-strap antennas at D, E-port (D and E antennas) are operated at 80.5 and $80 \mathrm{MHz}$ respectively in dipole phasing $(0, \pi)$. In the experiments reported here, the 4-strap antenna at J-port ( $\mathrm{J}$ antenna) was operated at $50 \mathrm{MHz}$ in $(0, \pi, \pi, 0)$ (symmetric or heating), $(0, \pi / 2, \pi,-\pi / 2)$ and $(0,-\pi / 2, \pi, \pi / 2)$ (current drive) phasings. For J antenna, the strap width, separation (center to center) and major radius location are $8 \mathrm{~cm}, 26.6 \mathrm{~cm}$ and $93.5 \mathrm{~cm}$ respectively. This leads to $k_{\|}=10 \mathrm{~m}^{-1}\left(n_{\phi}=7\right)$ at the magnetic axis for the peak of the radiated spectrum for symmetric phasing, and $k_{\|}=9 \mathrm{~m}^{-1}\left(n_{\phi}=6\right)$ for current drive phasing. Since poloidal field effects modify and can potentially reverse the $k_{\|}$spectrum of the mode converted waves, labeling the two current drive phasings co- and counter-current drive as in the usual practice is not straightforward for MCCD scenarios. In general, the MCCD direction should depend on the direction of the toroidal magnetic field and ohmic plasma current, shown for C-Mod on Fig. 1. For clarity, we will denote the antenna phasings according to the predicted current directions for the launched antenna spectrum, ignoring poloidal field effects. Using this convention, the $(0, \pi / 2, \pi, 3 \pi / 2)$ $\left(+90^{\circ}\right)$ and $(0,-\pi / 2,-\pi,-3 \pi / 2)\left(-90^{\circ}\right)$ phasings are called counter- and co-current drive respectively.

In the experiments reported here, the plasma mix was $\mathrm{D},{ }^{3} \mathrm{He}$ and $\mathrm{H}$, with a 
central electron density $n_{e} \sim 1.5 \times 10^{-20} \mathrm{~m}^{-3}$. ${ }^{3} \mathrm{He}$ gas was injected around $200 \mathrm{~ms}$ after breakdown in deuterium in order to obtain the desired ${ }^{3} \mathrm{He}$ concentration in the flattop phase $\left(0.5-1.5 \mathrm{sec}\right.$ here). A residual hydrogen content $n_{H} / n_{e} \sim 5-10 \%$ is due to wall recycling and can be estimated for the ratio of $H_{\alpha}$ to $D_{\alpha}$ emission at the plasma edge. At a toroidal field of $5.3 \mathrm{~T}$, a ${ }^{3} \mathrm{He}$ concentration $n_{3} \mathrm{He} / n_{e}=20 \%$ and $\mathrm{H}$ concentration $n_{H} / n_{e}=5 \%$ creates conditions for mode conversion on the magnetic axis at $50 \mathrm{MHz}$. In this scenario, the ion cyclotron layers for ${ }^{3} \mathrm{He}$ and deuterium are also present in the plasma. The small hydrogen concentration also allows $\mathrm{D}(\mathrm{H})$ minority heating on axis with the E antenna at $80 \mathrm{MHz}$. Figure 2 shows the location of the layers at $50 \mathrm{MHz}$ and the plasma shape for the two sets of discharges 1050729 and 1050802, which will be analyzed in detail in this paper. The location of the mode conversion surface depends mostly on the magnetic field and the ${ }^{3} \mathrm{He}$ concentration. Increasing the ${ }^{3} \mathrm{He}$ concentration moves the mode conversion layer away from the ${ }^{3} \mathrm{He}$ cyclotron layer towards the high field side, while increasing the magnetic field moves the cyclotron and mode conversion layers towards the low field side.

The radial location of the mode conversion layer can be estimated experimentally from the electron deposition profiles. In this experiment, the profiles are obtained using fast turn-offs in the ICRF power $(\leq 10 \mu s)$ and a break-in-slope approach [Gambier 90, Lin 03]. The derivative of the electron temperature $\frac{\partial T_{e}(r)}{\partial t}$ is determined immediately before and after the turn-off by fitting the slope of $T_{e}(t)$ in a time interval $\delta t$. From the difference, the electron heating power density $S(r, \delta t) \approx \frac{3}{2} n_{e} \Delta \frac{\partial T_{e}(r)}{\partial t}$ associated with RF absorption can be calculated at the location of the temperature measurement.

On Alcator C-Mod, the break-in-slope approach can be routinely used with temperature measurements from Electron Cyclotron Emission (ECE) diagnostics. The experiments reported here used a 10-channel grating polychromator (GPC) [O'Shea 97] and a 32 channel 2nd harmonic X-mode radiometer (FRC-ECE) [Heard 99]. A $50 \mu \mathrm{s}$ time resolution was used in the analysis. The electron density is measured with a Thomson scattering system [Hughes 03]. The phase contrast imaging (PCI) system [Mazurenko 01] on C-Mod was not used in the experiment since the $\mathrm{q}=1$ surface is outside its observation window.

The location of the peak electron absorption from the break-in-slope approach can be used to estimate the ${ }^{3} \mathrm{He}$ concentration. The concentration for which the predicted radial location of the mode conversion surface matches the peak electron heating location can be considered as a quick estimate during the experiment. A more accurate estimate is obtained by comparing the measured deposition profiles and TORIC simulations with different ${ }^{3} \mathrm{He}$ concentrations. This approach constrains the $n_{3} \mathrm{He} / n_{e}$ within $\pm 1 \%$, and is generally within 2-3 percentage points of the initial estimate. In previous experiments, the estimated location was also shown to be consistent with the radial location of the density fluctuations measured with PCI. The location of the peak fluctuation amplitude measured by PCI changes only by 1 or $2 \mathrm{~cm}$ in the flattop.

Since the changes in the ${ }^{3} \mathrm{He}$ concentration between or during discharges are small, a controlled sweep of the mode conversion layer can be obtained by a slow ramp of the 
toroidal field.

\section{Sawtooth period changes with MCCD}

This section presents and discusses experimental data for two sets of discharges in which the mode conversion layer was swept partially or completely through the inversion radius surface on the high field side.

\subsection{Experimental data}

The evolution of the toroidal field and electron temperature measured by the FRC-ECE $\mathrm{X}$-mode radiometer is shown on Fig. 3 for the two sets of discharges 1050802 (a) and 1050729 (b). The temperature trace corresponds to a single ECE channel. Therefore the location of the temperature measurement changes as the toroidal field is varied, but it remains inside the $\mathrm{q}=1$ surface and allows a comparison of the sawtooth cycle and its period. A comparison of the sawtooth period evolution is shown on Figs. 4 and 5 . In the two sets of discharges 1050729 and 1050802, the antenna phasing was the only control parameter varied.

Significant differences in the sawtooth period evolution are observed in both sets of discharges. Comparing Figs. 4 and 5, it can be seen that as the mode conversion layer moves away from the magnetic axis, the sawtooth period shortens to $\sim 4 \mathrm{~ms}$ in co-current drive then lengthens to $\sim 10 \mathrm{~ms}$. A reversed evolution is obtained for counter-current drive, although the period is not reduced as much. The sawtooth period lengthens to $11 \mathrm{~ms}$. The evolution in symmetric phasing is similar to that in co-current drive phasing until $0.9 \mathrm{~s}$ (see Fig. 4). Using concentration estimates from the break-in-slope analysis, it is possible to compare the location of the mode conversion surface with the inversion radius. In the 1050802 discharges, the inversion radius is around $75.5 \mathrm{~cm}$. This is about $1 \mathrm{~cm}$ outside the mode conversion layer location for which the the co and counter-current drive curves crossover. In the 1050729 discharges, only half of the evolution is obtained but the cross-over position is less obvious. The curves suggest it occurs when the mode conversion layer is around $75 \mathrm{~cm}$. Since the inversion radius is around $76.5 \mathrm{~cm}$ in this discharge, we find that the relative positions are qualitatively consistent.

While the achieved discharges parameters are very similar, unexpected events like impurity injections or antenna faults can hinder the comparison. Such events are highlighted with shaded color in Fig. 3. Impurity injections induce a rapid increase in the radiated power and cool down the edge plasma. The core temperature is reduced and the sawtooth period is typically shortened. The effects typically last for a confinement time $\tau_{E} \sim 50 \mathrm{~ms}$, after which the plasma recovers. This typical evolution after an impurity event is illustrated on Fig. 5 between $0.95-1$ s. Similar events occurred in the counter-current drive discharge of 1050802 around $0.75 \mathrm{~s}$, and repeatedly in the heating discharge of 1050802 after 0.9 s. In addition to the transient effect on temperature, it is possible that the impurity influx changes the plasma composition and therefore the 
location of the mode conversion layer, although this effect is hard to evaluate.

\subsection{Initial discussion of current drive effects}

The observed variations in the sawtooth period are similar to that obtained in ICCD or Electron Cyclotron Current Drive (ECCD) experiments when the deposition is scanned around the $\mathrm{q}=1$ surface. In [Bhatnagar 94], as the ion cyclotron layer was swept on JET through the inversion radius $r_{i n v}$ on the inboard side, $+90^{\circ}$ injection inside $r_{i n v}$ shortened the sawtooth period and lengthened it outside $r_{i n v}$. The trend was reversed with $-90^{\circ}$ injection. On the outboard, the changes were in the opposite direction in both phasings. The observed sawtooth period evolution was attributed to local changes in the safety factor shear at the $\mathrm{q}=1$ surface due to localized ICCD. Recent experiments with ECCD on ASDEX-Upgrade [Mueck 05] also have also provided evidence for this mechanism. As the ECCD deposition was scanned across the plasma radius, sawtooth oscillations were completely stabilized with co-ECCD outside the inversion radius or counter-ECCD inside the inversion radius. Complete stabilization is usually the equivalent of a long or infinite sawtooth period. The sawtooth period can also be decreased with co-ECCD inside the $r_{i n v}$. In further experiments on ASDEX-Upgrade [Manini 05], the sawtooth period evolution was studied in a similar scan but without complete stabilization and using different current profile widths. Again, the sawtooth period was lengthened for co-current drive outside the inversion radius, and shortened inside. The evolution was reversed in counter-current drive. Note that in both ASDEX-Upgrade experiments, the plasma were also heated with Neutral Beam Injection (NBI). Careful analysis was required to isolate the ECCD and NBI-related contributions to the sawtooth period changes.

These results suggests that localized MCCD could account for the observed sawtooth period evolution in the experiments reported here. The location of the inversion radius and mode conversion layer is consistent and so is the evolution for the opposite phasings. However, it is essential to discuss other possible mechanisms which may affect the sawtooth period and their relevance to this experiment. Since the plasmas considered here are ICRF-heated with exclusion of other auxiliary heating systems, the main competing processes are related to fast wave ion cyclotron damping and fast ions populations.

The stabilizing effect of energetic ions inside the $q=1$ surface is discussed in [Porcelli 91] and has been obtained experimentally with ICRF minority heating on several tokamaks including C-Mod. Varying the antenna phasing can change the fast particle pressure profiles inside the $\mathrm{q}=1$ surface due to an ICRF-induced particle pinch [Eriksson 98] and thus result in sawtooth period changes. In the experiment reported here, minority heating of ${ }^{3} \mathrm{He}$ ions can occur in addition to $\mathrm{MECH}$, and while the large ${ }^{3} \mathrm{He}$ concentration minimizes this mechanism, it is not possible to exclude completely a contribution from an energetic ${ }^{3} \mathrm{He}$ ion population. But this contribution alone cannot account for the observed variations. Indeed, a comparison between the ion cyclotron 
layer positions in the 1050729 and 1050802 discharges on Fig. 2 shows that at the cross-over point the ${ }^{3} \mathrm{He}$ ion cyclotron layer was on axis and around the inversion radius respectively. In the 1050802 discharges, the ${ }^{3} \mathrm{He}$ ion cyclotron layer is inside the inversion radius surface after $0.8 \mathrm{sec}$, yet the sawtooth period in the three phasings is comparable after $1.1 \mathrm{sec}$. The scan was continued until $1.4 \mathrm{sec}$, at which point the ${ }^{3} \mathrm{He}$ ion cyclotron layer is on axis and the sawtooth period (not shown) is still unchanged as the antenna phasing is varied. The sawtooth period is also only slightly longer than during the ohmic phase (before .6 sec), indicating little fast particle stabilization. Therefore, a contribution from energetic ${ }^{3} \mathrm{He}$ ions in this experiment is very unlikely.

Following the same approach, one can exclude ICCD as a dominant mechanism in this experiment. While the ${ }^{3} \mathrm{He}$ ion cyclotron layer is near the inversion radius in the 1050802 discharges, it is on axis for the 1050729 discharges and ICCD cannot be possibly involved. Ion Cyclotron Current Drive has been investigated on Alcator C-Mod with $\mathrm{D}(\mathrm{H})$ minority heating but a similar deposition scan as discussed here did not induce clear changes in the sawtooth period. As pointed out in [Bhatnagar 94], the current drive efficiency for $\mathrm{D}\left({ }^{3} \mathrm{He}\right)$ scenario is lower compared to $\mathrm{D}(\mathrm{H})$. Therefore, comparing these $\mathrm{D}(\mathrm{H})$ minority heating results with the $\mathrm{D}\left({ }^{3} \mathrm{He}\right)$ mode conversion regime here, one may conclude that ICCD is not the dominant contribution here.

Based on this initial discussion, it appears that localized current drive around the mode conversion surface is the most plausible mechanism to explain the observed sawtooth period evolution. To further support this conclusion, we will show in the next section that the driven current profiles predicted by full wave TORIC simulations are qualitatively consistent with the experimental results. The evolution in heating phasing will be discussed separately in the last section.

\section{TORIC modeling}

\subsection{Presentation of the TORIC code}

The full wave code TORIC [Brambilla 99] solves the finite Larmor radius (FLR) equations in the Ion Cyclotron Range of Frequencies for axisymmetric plasmas. The $\mathrm{RF}$ electric field solution on each flux surface $\psi$ is represented at a given frequency as a sum of poloidal and toroidal modes:

$$
\vec{E}(\psi, \theta, \phi)=\sum_{n_{\phi}} \exp \left(i n_{\phi} \phi\right) \sum_{m_{\theta}} \tilde{E}_{\psi, m_{\theta}, n_{\phi}} \exp \left(i m_{\theta} \theta\right)
$$

The sum is truncated at specified poloidal and toroidal mode numbers $m_{\theta}$ and $n_{\phi}$, where the limits are chosen so that the wavefields of the excited ion cyclotron waves can be numerically resolved. The coefficients $\tilde{E}_{\psi, m_{\theta}, n_{\phi}}$ are calculated by substituting the electric field solution in the wave equation and using a variational approach. The toroidal symmetry allows to calculate each $n_{\phi}$ solution independently. However, the poloidal modes and radial solutions are coupled in general due to geometric and finite Larmor radius effects. The limits assumed in TORIC for the plasma response simplify 
the resulting system of equations significantly, allowing for an efficient numerical solution with a sparse, block tridiagonal stiffness matrix. Still, most of the relevant physics around the cyclotron frequencies of the plasma ions can be retained. The plasma model describes the compressional, torsional Alfvén branches, including kinetic effects, and the lowest order Ion Bernstein Waves (IBW), absorption by ions at the cyclotron frequency and its first harmonics and by electrons through electron Landau and transit time damping. The leading effects of toroidicity on cyclotron absorption and parallel dispersion are also taken into account. The electron Landau damping of Ion Bernstein Waves is treated by adding an imaginary part to the FLR coefficient to include relevant contributions from higher order terms in $k_{\perp} \rho_{i}$.

The simulations presented here are done with a parallelized version of the code, run on a 48-CPU cluster at the MIT Plasma Science and Fusion Center. Typically, 500 radial elements and 127 to 255 poloidal modes are required to obtain convergence for typical mode conversion scenarios in C-Mod. The antenna spectrum can be calculated using the one-dimensional FELICE code or simplified antenna models in TORIC. While the latter option was used for the PCI analysis in [Lin 05], we used FELICE in this paper since it provides a better description of the antenna geometry and of the density profiles at the plasma edge. For the C-Mod antennas, both approaches predict that negligible power is coupled to modes with $\left|n_{\phi}\right| \geq 15$ and the code is usually run with $-20 \leq n_{\phi} \leq 20$ for C-Mod scenarios. A complete simulation of a mode conversion scenario is run in less than a day at this resolution, allowing a thorough and accurate survey of experimental cases.

\subsection{TORIC simulations}

TORIC simulations for the 1050802 discharges at $0.85 \mathrm{sec}$ are shown on Fig. 6 for the three phasings in the experiment. Fig. 6 (a) shows the power deposition profiles for electrons and all ions species. Electron absorption dominates and accounts for $\sim 60$

$\%$ of the coupled ICRF power. Over the entire antenna spectrum $\left(-20 \leq n_{\phi} \leq 20\right)$, the profiles show a two peak structure corresponding to $n_{\phi}= \pm 7$ at the maximum of the radiated spectrum. The co and counter-CD phasings favor positive or negative toroidal mode numbers respectively, while the symmetric heating phasing appears as a combination of the two. It appears that the power deposition is closer to the axis for positive $n_{\phi}$, which as we see later corresponds to a vertical shift in the deposition location along the mode conversion surface.

The current drive efficiency associated with each $\left(\psi, m_{\theta}, n_{\phi}\right)$ mode in the TORIC simulations can be calculated using the Ehst Karney parametrization [Ehst 91]. This allows to evaluate the reduction in the driven currents associated with magnetic trapping. Fig. 6 (b) shows an estimate of the current profiles for the 1050802 discharges based on the current profile reconstructed by EFIT and the driven currents computed by TORIC. For this off-axis mode conversion current drive scenario, the overall current drive efficiency is low. The net driven currents are less than $20 \mathrm{kA}$. While this value 
is small compared to the plasma current $I_{p}=600 k A$, the local change in the current density is nevertheless significant. The peak driven current density for the co-current drive phasing is $4 \mathrm{MA} \mathrm{m}^{-2}$. At the location of the mode conversion layer, the current density estimated by EFIT is $\sim 10 \mathrm{MA} \mathrm{m}^{-2}$. Therefore, we can expect significant changes in the q profile associated with these driven current profiles.

The shape of the driven current density profiles predicted by TORIC exhibits noticeable differences for the co, counter-CD and heating phasings. While the driven current density profile for co-current drive is peaked with a slight reversal outside, the profile in counter-current drive is bipolar, with currents in the counter-CD direction inside and in the co-direction outside. Note that the small difference in current density for $r / a<0.2$ corresponds to a weak FWCD effect.

With these simulation results, we can move forward in our interpretation of the experimental results. The driven currents predicted by TORIC are clearly large enough to change the safety profile locally. We can compare the overall shape of the driven current profiles predicted by TORIC with that in the other experiments mentioned above. In co-current drive phasing, the driven current profile is similar to that in ECCD experiments [Manini 05]. It increases the shear at the $q=1$ surface when deposited inside and decreases it when deposited outside, which results in an shorter sawtooth period inside and longer outside. The bipolar profile for counter-current drive phasing appears more similar to that in the ICCD experiments [Bhatnagar 94]. The driven currents tend to flatten the shear over a relatively large radius and thus lengthen the sawtooth periods. As in [Bhatnagar 94], the sawtooth period is not reduced during the sweep. In both cases, assuming that the $q=1$ surface does not move during the scan, the width of the predicted driven current profiles should also be consistent with the radial extent of the sawtooth period changes on Fig. 4. We can see that this is indeed the case in the co- and counter-current drive phasings.

Therefore, this qualitative comparison shows that the TORIC predictions and the experimental results are compatible. This supports the interpretation of the observed sawtooth period evolution in co- and counter-current drive phasing in terms of localized mode conversion current drive.

\section{Up-down asymmetries and evolution in heating phasing}

\subsection{Mode conversion physics and up-down asymmetries}

This overall agreement gives some confidence that the current drive predictions from TORIC may be correct. Since the shape of the driven current profiles is very asymmetric, it may be useful to give a brief physical interpretation for the underlying mode conversion physics. As mentioned in the introduction, the presence of a small poloidal field component can induce large upshifts in $k_{\|} \approx \frac{n_{\phi}}{R}+\frac{m_{\theta}}{r} \frac{B_{\theta}}{B}$. For off-axis mode conversion scenarios, the poloidal field term dominates. Since the propagation vector for mode converted Ion Bernstein Waves (IBW) and Ion Cyclotron Waves (ICW) points towards 
the low field side, the sign of the poloidal mode number $m_{\theta}$ is reversed above and below the midplane and so is therefore the direction of toroidal propagation for the mode converted waves. Mode converted waves damping above the midplane will drive current in one direction with respect to the plasma current, while mode converted damping below the miplane will drive current in the opposite direction. The direction and location of the current drive effects reflects therefore vertical asymmetries in the electron power deposition along the mode conversion surface. Fig. 7 shows a two-dimensional contour plot of the electron absorption for the TORIC simulations above in the counter-current drive case. The flux surfaces correspond to increments of $0.1 \mathrm{in} r / a$ mapped on the outer midplane, so that we can compare this plot with the counter-current drive curve on Fig. 6 (a). The deposition on the inside occurs above the midplane and induces counter-current drive, the deposition on the outside occurs below the midplane and induces co-current drive. For co-current phasing, the contour plot for the power density deposited on electrons is similar, however absorption below the midplane dominates and the driven currents are preferentially in the co-direction. Studies of the dispersion curves in a one-dimensional slab model [Parisot 05] suggest that the peak absorption occurs where the local poloidal field becomes large enough for mode conversion to ICW to start dominating over mode conversion to IBW. In this region the two waves cannot be clearly distinguished based only on the local dispersion relation. However, TORIC predicts little current drive from Ion Bernstein Waves away from the confluence region, therefore we can state that the MCCD currents in TORIC are driven by mode converted Ion Cyclotron Waves. The up-down asymmetry in the wavefields and power absorption close to the IBW-ICW confluence region cannot be explained on the basis of the local dispersion relation only. It has been observed almost systematically for C-Mod plasmas in two dimensional full wave simulations with TORIC, in particular during the comparison with PCI measurements [Lin 05], and with AORSA in the context of flow drive studies [Jaeger 03].

Perhaps the most striking consequence of this up-down asymmetry is the prediction of significant net current drive in symmetric phasing. As can be seen on Fig. 6 (b), the profile in symmetric phasing appears as an average over the current profiles in coand counter-current drive. However, because of the asymmetry in the two profiles, net currents in the co-direction are still present, with a peak current density of $1.5 \mathrm{MA}$ $\mathrm{m}^{-2}$. This can be again related to larger electron absorption at the IBW-ICW confluence below the midplane than above the midplane. In this context, an experimental study of the sawtooth period evolution in heating phase may give indications of this effect and constitute a further test of the TORIC MCCD modeling.

\subsection{Evolution in heating phasing}

The sawtooth period evolution for the heating phasing was studied as part of the 1050802 set of discharges. The evolution can be seen on Fig. 4. While impurity injections hamper the interpretation after $.9 \mathrm{sec}$, the earlier part appears to be similar to the evolution in co- 
current drive phasing case. This appears to be consistent with the TORIC predictions. However another effect can be important in this case and must be considered.

In addition to current drive, localized electron heating around the $q=1$ surface can affect the sawtooth period. This effect has been identified most clearly in Electron Cyclotron Heating (ECH) experiments on TCV [Angioni 03]. In these experiments, an ECH beam was injected in the plasma so that $k_{\|} \approx 0$. As the deposition location was swept through the inversion radius, the sawtooth period increased from $4 \mathrm{~ms}$ to $8 \mathrm{~ms}$ then decreases again to $4 \mathrm{~ms}$. This result can still be understood in terms of changes in the safety factor shear $s_{1}$ at the $\mathrm{q}=1$, however the process is less direct for localized current drive. As discussed in [Graves 05], the evolution of $s_{1}$ is related to the resistive diffusion of the poloidal field after a sawtooth reconnection event. As the resistivity gradient at the $\mathrm{q}=1$ surface is decreased due to localized heating just outside the $\mathrm{q}=1$ surface, the rise of the shear $s_{1}$ is slower. According to the Porcelli model [Porcelli 96], a sawtooth crash is triggered if $s_{1}$ exceeds a critical value $s_{c}$. Therefore, assuming that other possible trigger conditions are not met, localized heating outside the $q=1$ surface will delay the sawtooth crash and increase the period. The basis for this mechanism was confirmed through simulations with the transport code PRETOR [Boucher 92], using the trigger conditions in the Porcelli model. The code could reproduce qualitatively the sawtooth period evolution observed in the TCV experiments. As discussed in [Angioni 03], current drive with RF waves is always accompanied by localized heating, and therefore the effect discussed here will be present to some degree in current drive phasings. If the current drive effect can be considered symmetric with respect to the $\mathrm{q}=1$ surface, localized heating will induce an asymmetry and reinforce the co-current drive effect. The sawtooth period increase will be larger for co-current drive outside than for counter-drive inside. The decrease in the sawtooth period for counter-current drive outside the $\mathrm{q}=1$ surface will also be reduced. This behavior is in fact consistent with the observed sawtooth period evolution in C-Mod, which suggests that this effect may be involved.

Simulations similar to that with PRETOR for the TCV experiments would be necessary to estimate the strength of the heating effect on C-Mod. Assuming that resistivity gradient change is the dominant mechanism involved, we can argue that its contribution will be small in the experiments presented here. Indeed, strong profile stiffness for the electron temperature has been systemically observed in C-Mod [Greenwald 97] in almost all conditions, including the L-mode confinement regime here. Accordingly, the equilibrium electron temperature gradient and therefore resistivity gradient are expected to be largely unchanged as localized heating is applied. The observed profile resiliency is understood theoretically as due to turbulent transport by Ion Temperature Gradient (ITG) modes. In C-Mod, the electron and ion channels are tightly coupled due to high density at which the tokamak operates. The thermal equilibration is fast and maintains $\frac{T_{e}}{T_{i}} \approx 1$. Both ion and electron temperature gradient is thus determined by marginal stability for the ITG modes in the ion channel. This means that, in equilibrium, the temperature gradients remains close to the critical value 
for the onset of the turbulence. Of course, sawtooth reconnection implies a transient deviation from transport equilibrium inside the mixing radius, which means that the temperature gradients will be below the critical value for at least part of the sawtooth ramp phase. The $q=1$ surface is however close to the inversion radius and the local change in temperature during a sawtooth crash is small. Therefore we expect that marginal stability for turbulence will be restored quickly after sawtooth crashes. As a result, when the mode conversion heating surface moves outside the $q=1$ surface, the resistivity gradient at the $\mathrm{q}=1$ radius will not change significantly. Therefore localized heating is not expected to induce large changes in the sawtooth period.

On the other hand, very small driven currents can change the sawtooth period significantly, as stated in [Angioni 03]. This is confirmed by ECH experiments on TCV [Henderson 01], where small amounts of currents can be driven by the beam even for injection with $k_{\|} \approx 0$ due to upshifts associated with the field helicity. The small driven current density results in sawtooth period changes, in addition to that induced by localized heating. In the experiments, the beam launched with zero toroidal angle was swept through the entire plasma cross section and crossed the $q=1$ surface both above and below the midplane. A different sawtooth period evolution was observed in the two crossings for off-axis deposition, which could be be explained by localized heating effects alone. Ray tracing simulations indicated that small currents, with a total of $\sim 5 k A$ out of a plasma current $\sim 200 k A$, were driven with different polarity above and below the midplane due to poloidal field effects. This was confirmed by reversing the sign of the toroidal field and observing a mirrored behavior during the scan. The

authors concluded that small amounts of currents driven by the ECH were sufficient to change the sawtooth period significantly as compared to the effect of localized heating.

While this discussion is qualitative, it suggests that the localized electron associated associated with mode conversion may not be sufficient to explain the sawtooth period evolution in heating phasing on C-Mod. On the other hand, TORIC simulations indicate that significant MCCD currents can be driven in symmetric phasing. By comparing the magnitude of the driven currents and the sawtooth period changes in the three phasings, we conclude that the currents predicted by TORIC in heating phasing are sufficient to explain the observed sawtooth period evolution in the experiment. This analysis indicates that the observed sawtooth period evolution in the C-Mod MCCD experiments here is qualitatively consistent with the TORIC predictions for current drive phasings but also for the results in heating phasing. This can be regarded as an experimental evidence for the asymmetry in the MCCD predicted by TORIC simulations.

\section{Conclusions}

Significant changes in the sawtooth period have been obtained experimentally on Alcator C-Mod with ICRF mode conversion around the $\mathrm{q}=1$ surface. As the mode conversion layer was swept through the inversion radius in co-, counter-current drive and heating phasing, the period varied from $3 \mathrm{~ms}$ to $12 \mathrm{~ms}$. While mechanisms like fast particle 
stabilization or Ion Cyclotron Current Drive cannot explain the experimental results, the differences in the sawtooth period evolution between co and counter-current drive phasing are consistent with changes in the shear at the $q=1$ surface associated with localized current drive. According to numerical simulations with the full wave TORIC code, currents comparable in magnitude to the local ohmic current density can be driven by mode converted Ion Cyclotron Waves. The shape of the predicted driven current profiles is consistent with the experimental results. A strong up-down asymmetry in the wave fields and power deposition is observed in the simulations, resulting in significant net co-current drive for symmetric antenna phasing. This prediction is consistent with the observed sawtooth period evolution in heating phasing, which is comparable to that in co-current drive phasing. Localized electron heating could also account for this similarity but it is unlikely to be important in the experiment since temperature profile stiffness prohibits large changes in the resistivity gradient at the $q=1$ surface. In summary, the sawtooth period changes observed experimentally in co, counter-current drive and heating phasing can be explained by the MCCD profiles predicted by TORIC. These results can therefore be regarded as an experimental indication for the asymmetry in the MCCD predicted in the simulations.

\section{Acknowledgments}

This work is supported by Department of Energy Coop. Agreement DE-FC0299ER54512 and utilized the MIT Plasma Science and Fusion Center Theory Group parallel computational cluster.

\section{References}

[Angioni 03] C. Angioni et al. Effects of localized electron heating and current drive on the sawtooth period. Nucl. Fusion, vol. 43, pages 455-468, 2003.

[Bhatnagar 94] V.P. Bhatnagar et al. Local magnetic shear control in a tokamak via fast wave minority ion current drive: theory and experiments in JET. Nucl. Fusion, vol. 34, page 1579, 1994.

[Bonoli 97] P. Bonoli et al. Electron heating via mode converted ion Bernstein waves in the Alcator C-Mod tokamak. Physics of Plasmas, vol. 4, page 1774, 1997.

[Boucher 92] D. Boucher \& P.H. Rebut. In Proc. IAEA Tech. Com. on Advances in Simulation and Modell. of Thermonuclear Plasmas, Montreal, page 142, 1992.

[Brambilla 99] M. Brambilla. Numerical simulation of ion cyclotron waves in tokamak plasmas. Plasma Phys. Cont. Fusion, vol. 41, pages 1-34, 1999.

[Ehst 91] D. Ehst \& C. Karney. Approximate formula for radio-frequency current drive efficiency with magnetic trapping. Nucl Fusion, vol. 31, page 1933, 1991.

[Eriksson 98] L-G. Eriksson et al. Evidence for a Wave Induced Particle Pinch in the Presence of Toroidally Asymmetric ICRF Waves. Phys. Rev. Lett., vol. 81, page 1231, 1998.

[Faulconer 89] D.W. Faulconer et al. The role of temperature and rotationnal transform in ICRH mode conversion. In Proc. 14th EPS Conf. on controlled fusion and plasma physics, volume 11D (ECA), page 932, 1989.

[Gambier 90] D.J. Gambier et al. ICRF power deposition profile and determination of the electron 
thermal diffusivity by modulation experiments in JET. Nuclear Fusion, vol. 30, pages 23-34, 1990.

[Graves 05] J. P. Graves et al. Sawtooth control in fusion plasmas. Plasma Phys. Control. Fusion, vol. 47, pages B121-B133, 2005.

[Greenwald 97] M. Greenwald et al. H mode confinement in Alcator C-Mod. Nucl. Fusion, vol. 37, page 793, 1997.

[Heard 99] J.W. Heard et al. High resolution electron cyclotron emission temperature profile and fluctuation diagnostic for Alcator C-Mod. Rev. Sci. Instr., vol. 70, pages 1011-1013, 1999.

[Henderson 01] M.A. Henderson et al. Poloidally asymmetric plasma response with ECH deposition near $q=1$ in TCV. Fus. Eng. Des., vol. 53, pages 241-248, 2001.

[Hughes 03] J.W. Hughes et al. Thomson scattering upgrades on Alcator C-Mod. Rev. Sci. Instr., vol. 74, pages 1667-1670, 2003.

[Hutchinson 93] I.H. Hutchinson et al. First results from Alcator-C-MOD. Phys. Plasmas, vol. 1, pages 1511-1518, 1993.

[Jaeger 03] E.F. Jaeger et al. Sheared Poloidal Flow Driven by Mode Conversion in Tokamak Plasmas. Phys. Rev. Lett., vol. 90, page 195001, 2003.

[Lin 03] Y. Lin et al. ICRF mode conversion electron heating in D-H plasmas in the Alcator C-Mod tokamak. Plasma Phys. Cont. Fusion, vol. 45, pages 1013-1026, 2003.

[Lin 05] Y. Lin et al. Observation and modelling of ion cyclotron range of frequencies waves in the mode conversion region of Alcator C-Mod. Plasma Phys. Control. Fusion, vol. 47, pages 1207-1228, 2005.

[Majeski 96] R. Majeski et al. Mode Conversion Heating and Current Drive Experiments in TFTR. Phys. Rev. Lett., vol. 76, pages 764-767, 1996.

[Manini 05] A. Manini et al. Optimisation of Sawtooth Control using ECCD in ASDEX Upgrade. In 32th EPS Conf. on Plasma Physics, Tarragona, ECA vol 29C, 2005.

[Mantsinen 04] M.J. Mantsinen et al. Localized bulk electron heating with ICRF mode conversion in the JET tokamak. Nucl. Fusion, vol. 44, pages 33-36, 2004.

[Mazurenko 01] A. Mazurenko. Phase Contrast Imaging on Alcator C-Mod. PhD thesis, MIT, Cambridge, MA, 2001.

[Mueck 05] A. Mueck et al. Sawtooth control experiments on ASDEX Upgrade. Plasma Phys. Cont. Fusion, vol. 47, pages 1633-1655, 2005.

[Nelson-Melby 03] E. Nelson-Melby et al. Experimental Observations of Mode-Converted Ion Cyclotron Waves in a Tokamak Plasma by Phase Contrast Imaging. Phys. Rev. Lett., vol. 90, page 155004, 2003.

[Noterdaeme 96] J-M. Noterdaeme et al. ICRF Heating results in ASDEX-Upgrade and W\%-AS. In Proc. 16th Conf. on Fusion energy, Montreal, CA, volume 3. IAEA, 1996.

[O'Shea 97] P.J. O'Shea. Measurements of ICRF Power Deposition and Thermal Transport with an ECE Grating Polychromator on the Alcator C-Mod Tokamak. PhD thesis, Mass. Inst. of Tech, Cambridge, MA, 1997.

[Parisot 05] A. Parisot et al. Numerical studies of poloidal field effects on ICRF Mode conversion. In Proceedings of the 16th Topical Conference on RF power in plasmas, Park City, UT, April 2005.

[Perkins 77] F.W. Perkins. Heating Tokamaks via the ion-cyclotron and ion-ion hybrid resonances. Nucl. Fusion, vol. 17, page 1197, 1977.

[Porcelli 91] F. Porcelli. Fast particle stabilisation. Plasma Phys. Cont. Fusion, vol. 33, pages 1601-1620, 1991.

[Porcelli 96] F. Porcelli et al. Model for the sawtooth period and amplitude. Plasma Phys. Control. Fusion, vol. 38, pages 2163-2186, 1996.

[Ram 91] A. K. Ram \& A. Bers. Propagation and damping of mode converted ion-Bernstein waves in toroidal plasmas. Phys. of Fluids B, vol. 3, pages 1059-1069, 1991. 


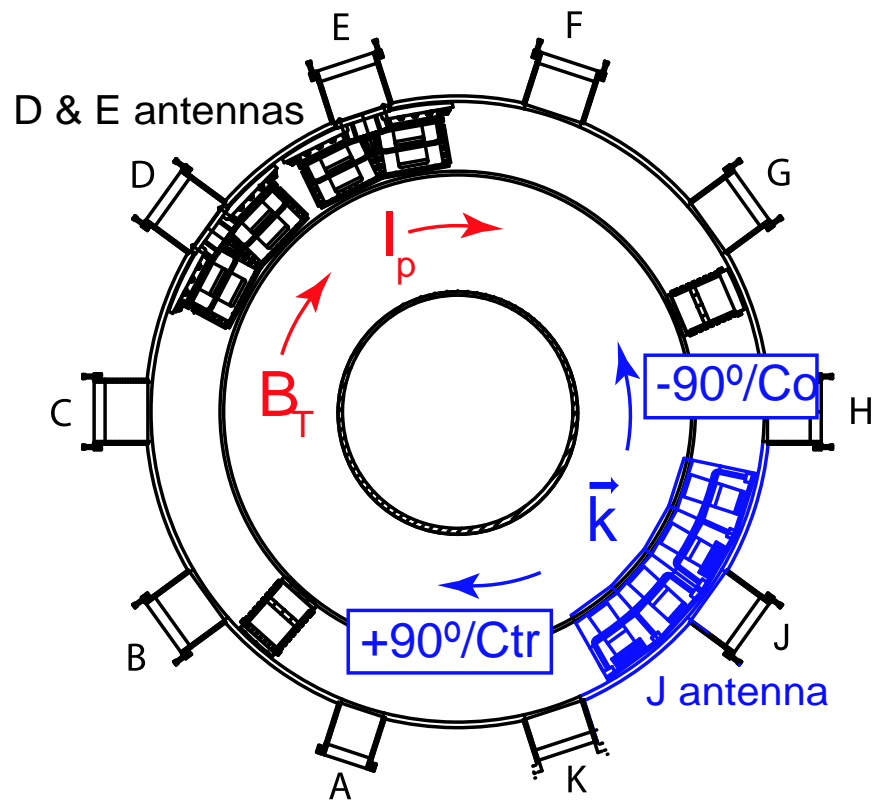

Figure 1. Top view of the Alcator C-Mod tokamak showing the three ICRF antennas and the directions of the magnetic field and plasma current. The denominations for the current phasings are also indicated, with the blue arrows showing the directions of the wave vector $\vec{k}$

[Saoutic 96] B. Saoutic et al. Mode conversion heating experiments on the Tore Supra Tokamak. Phys. Rev. Lett, vol. 76, page 1647, 1996.

[Stix 92]

[Swanson 85]

T. H. Stix. Waves in plasma. American Institute of Physics, 1992.

D.G. Swanson. Radio Frequency Heating in the Ion Cyclotron Range of Frequencies. Physics of Fluids, vol. 28, page 2645, 1985.

[Wilson 98]

J. R. Wilson et al. Ion cyclotron range of frequencies heating and flow generation in deuterium-tritium plasmas. Phys. plasmas, vol. 5, pages 1721-1727, 1998.

[Wright 04]

[Wukitch 02]

[Wukitch 05]

J.C. Wright et al. Full wave simulations of fast wave mode conversion and lower hybrid wave propagation in tokamaks. Phys. Plasmas, vol. 11, pages 2473-2479, 2004.

S.J. Wukitch et al. Performance of a compact four-strap fast wave antenna. In 19th Fusion Energy Conference, Lyon, France, IAEA FT/P1-14, 2002.

$\mathrm{S}$. Wukitch et al. Ion cyclotron range of frequency mode conversion physics in Alcator C-Mod: Experimental measurements and modeling. Phys. Plasmas, vol. 12, page 056104, 2005. 


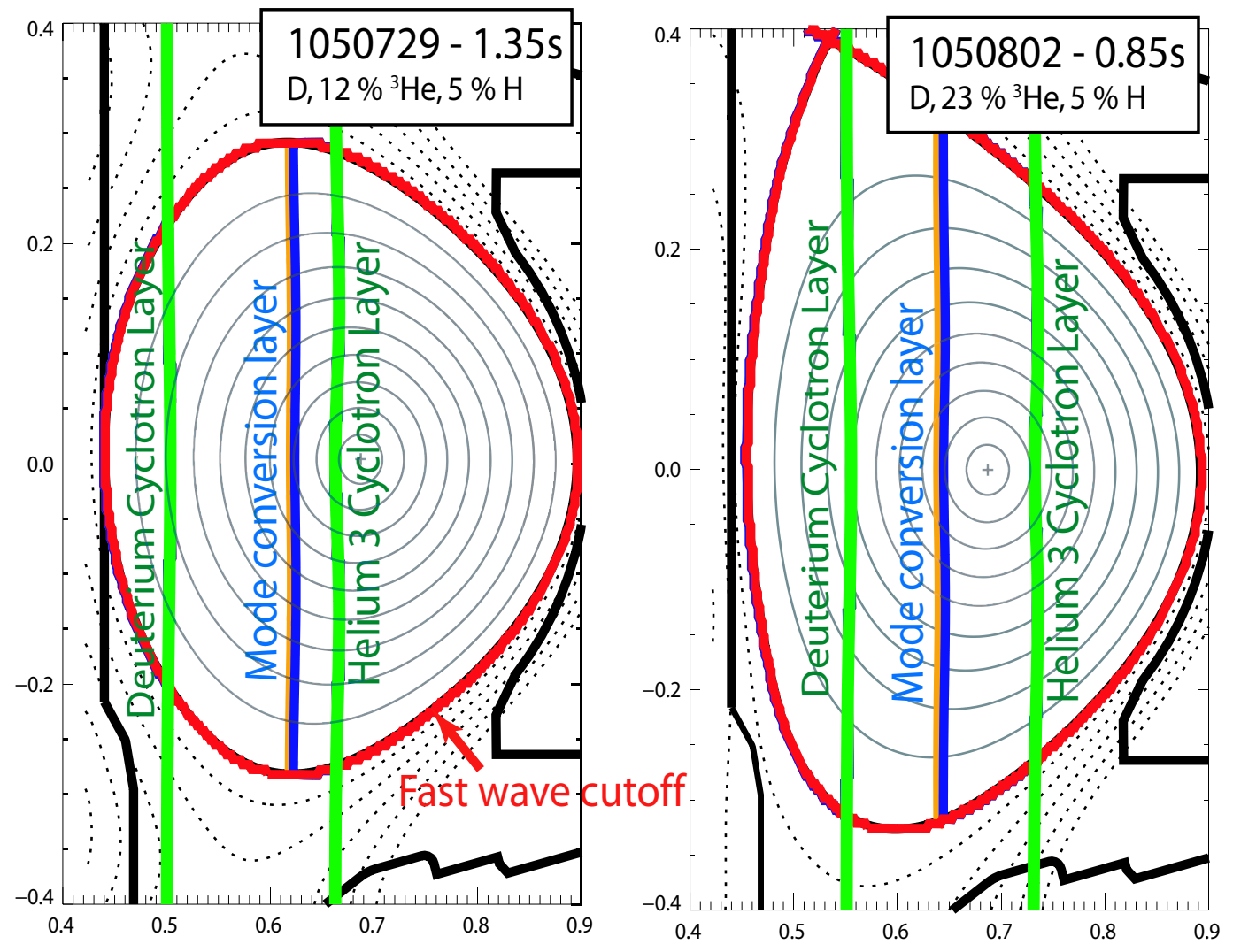

Figure 2. Magnetic configuration and position of the mode conversion and cyclotron layers for two sets of discharges. 
(a)

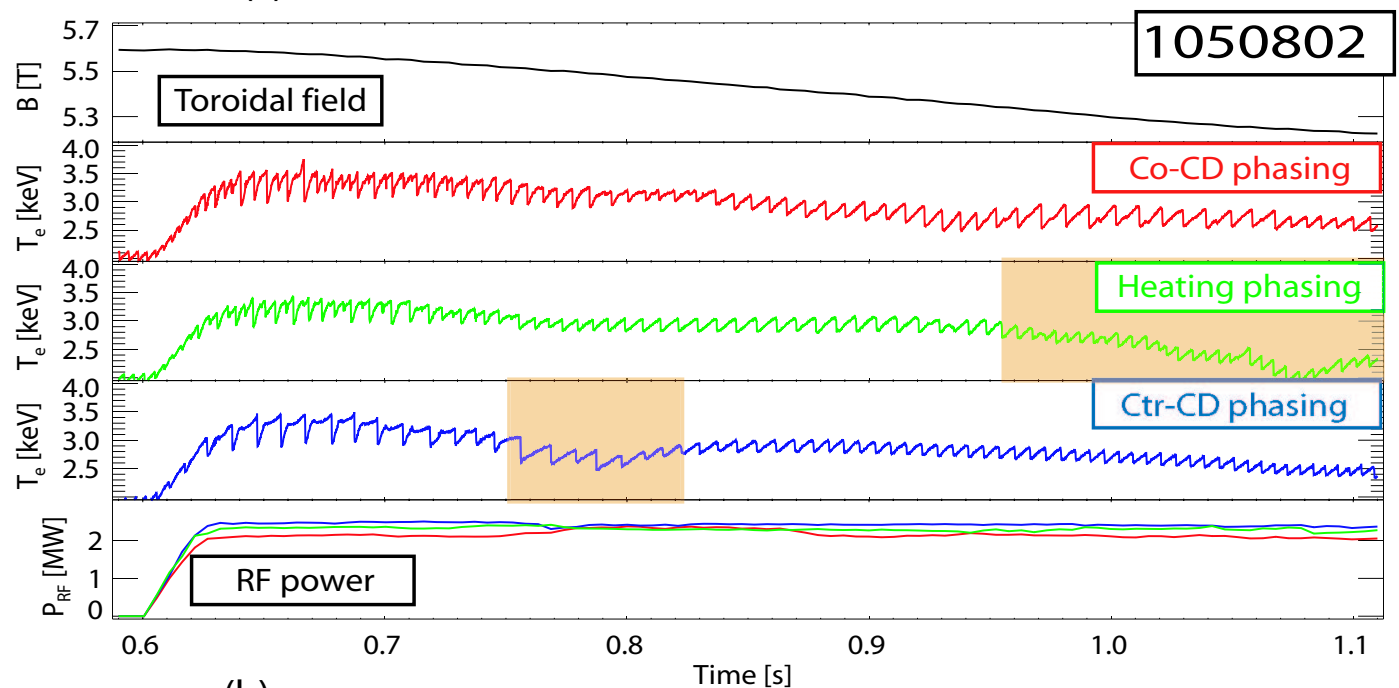

(b)

1050729

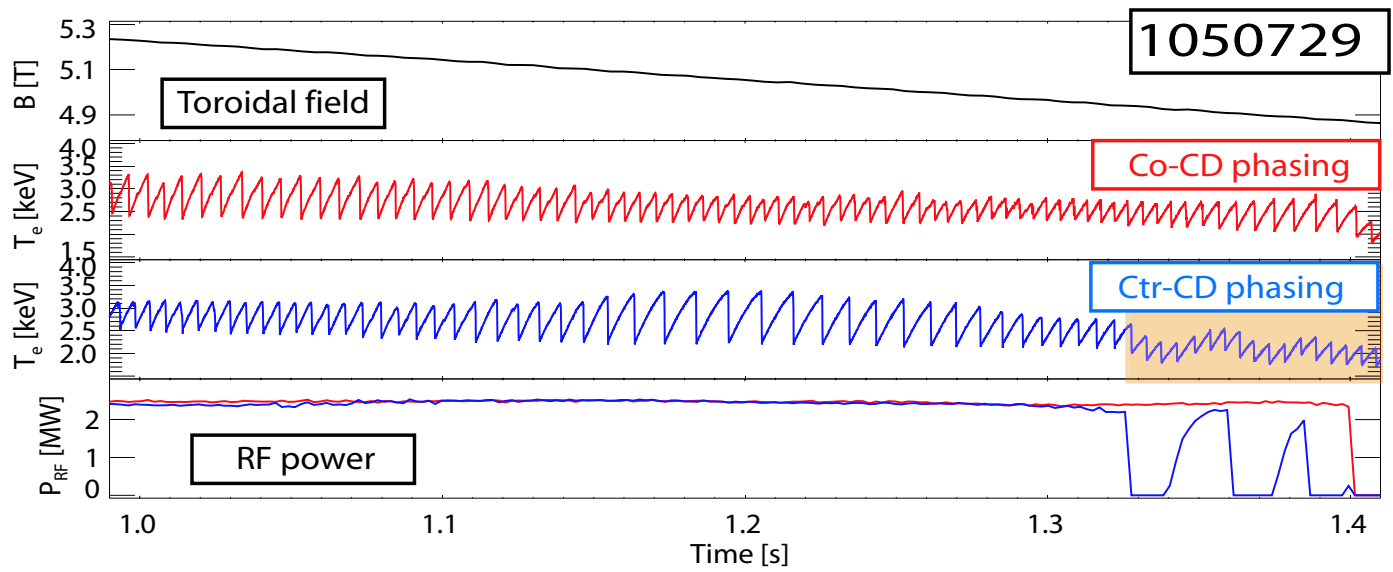

Figure 3. Evolution of the sawteeth in two groups of discharges with similar parameters but different phasings. The shaded regions indicate events like impurity injections or RF trips which hinder the comparison. 


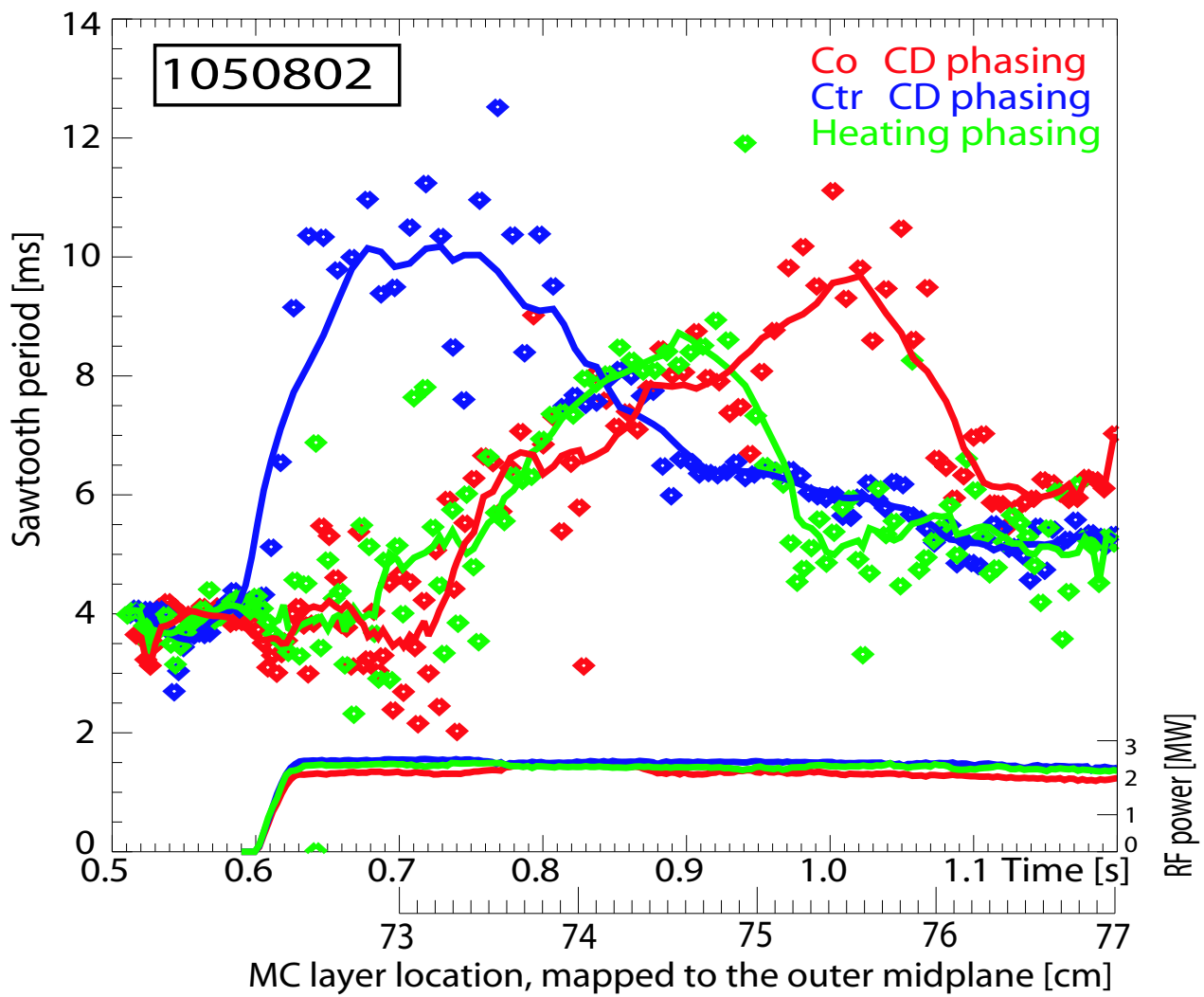

Figure 4. Evolution of the sawteeth in the first group of discharges on Fig. 3. The mode conversion layer location is deduced from the toroidal field and ${ }^{3} \mathrm{He}$ concentration estimate. The inversion radius is at about $75.5 \mathrm{~cm}$. 


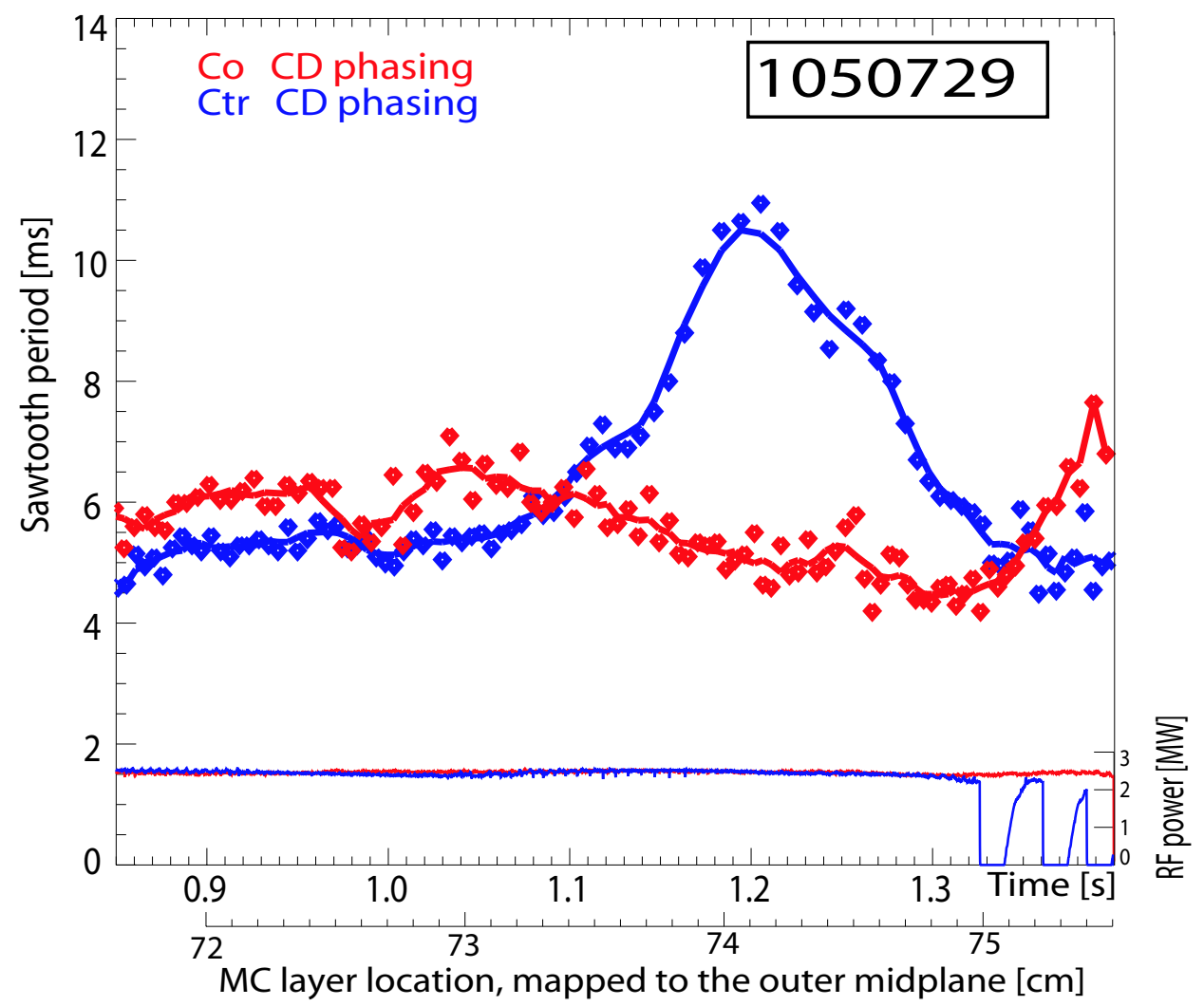

Figure 5. Evolution of the sawteeth in the second group of discharges on Fig. 3. The mode conversion layer location is deduced from the toroidal field and ${ }^{3} \mathrm{He}$ concentration estimate. The inversion radius is at about $76.5 \mathrm{~cm}$.
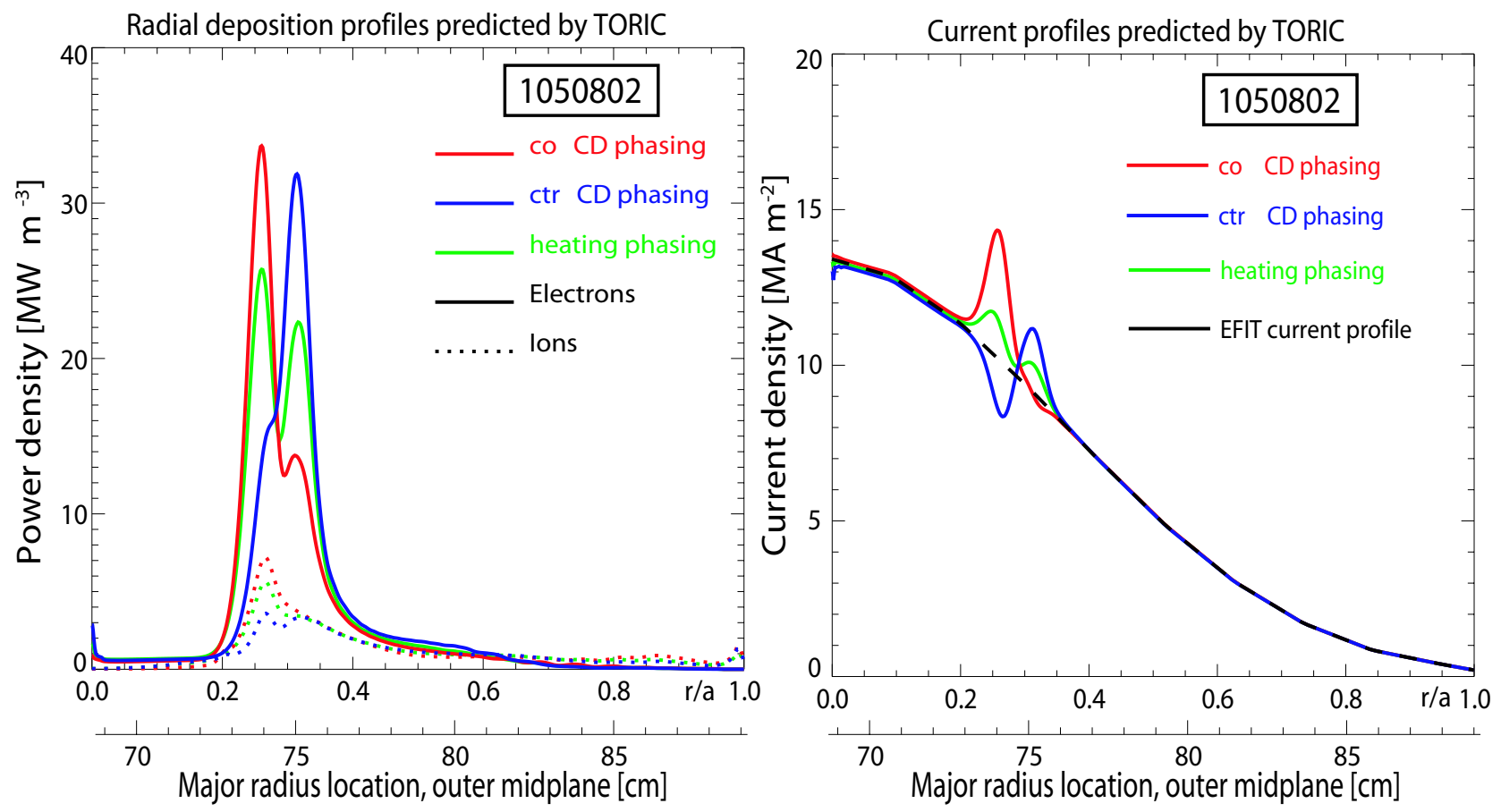

Figure 6. Power deposition and driven current profiles predicted by TORIC for the 1050802 discharges at $0.85 \mathrm{sec}$. 


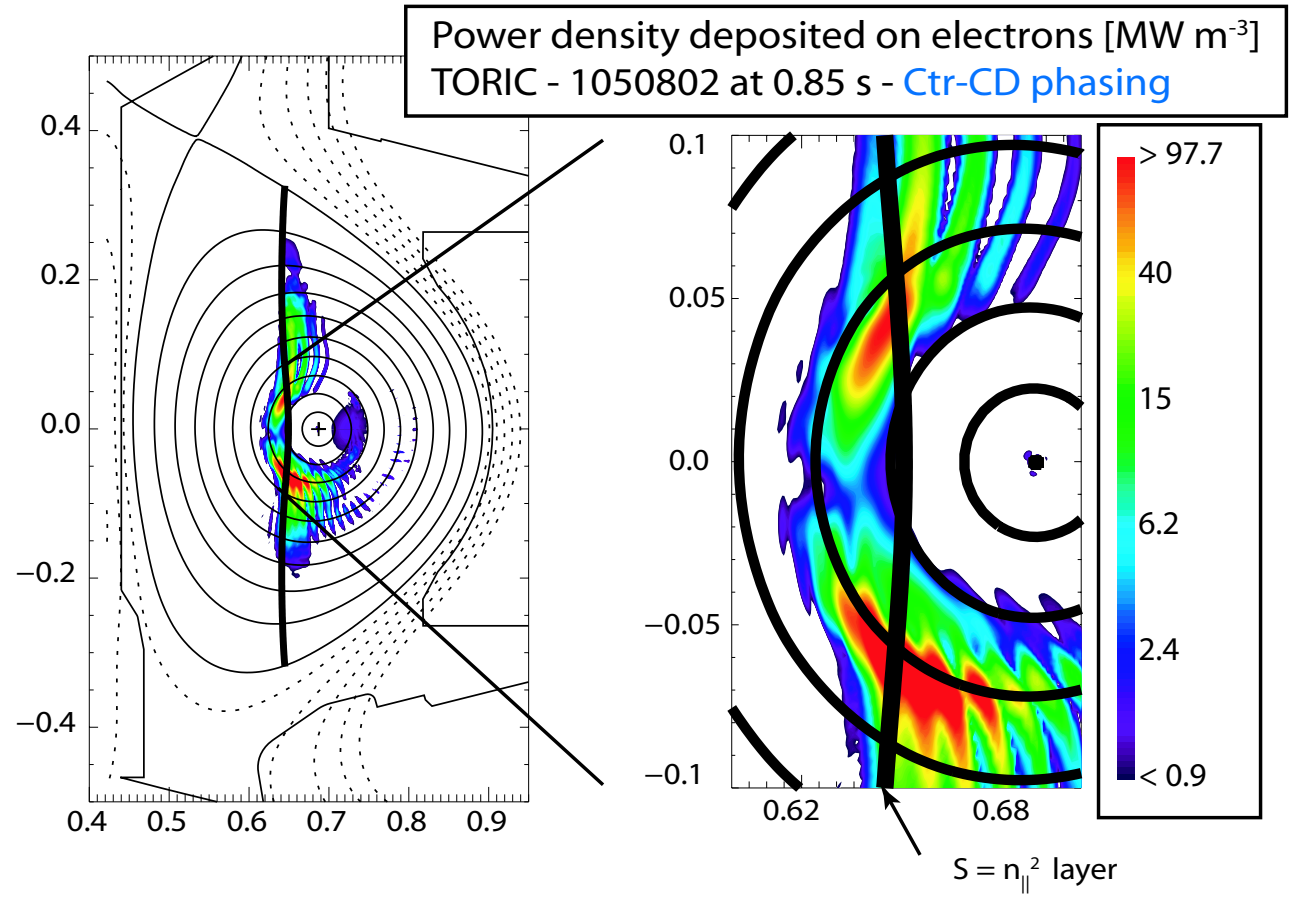

Figure 7. Two dimensional contour plot for the electron power absorption predicted by TORIC. This is for the 1050802 discharges at $0.85 \mathrm{sec}$. 